\title{
CURRENT RESEARCH ON ROCK ART AT GUA TAMBUN, PERAK, MALAYSIA
}

\author{
Noel Hidalgo Tan ${ }^{1}$ and Stephen Chia $^{2}$ \\ Center for Global Archaeological Research, University Sains, Malaysia; \\ ${ }^{1}$ seaarch@gmail.com, ${ }^{2}$ stephen@usm.my
}

\begin{abstract}
The rock art site of Gua Tambun in Perak, Malaysia was first reported by J. M. Matthews in 1959, following the discovery of the rock paintings by a British military officer. An estimate of more than 80 forms of animals, humans, geometric designs and many other indistinct and vague forms of paintings were reported on the walls of the rock shelter. Since then, no further in-depth research of the rock art has been reported, while time and weather have eroded and faded the paintings even more. In early January 2009, the site was revisited by the authors to document and to study the rock art in detail. The rock art was documented using a combination of closerange, high-resolution digital photography and digital image analysis to reconstruct and recompose the faded images. Samples of the material used for painting the rock art were also collected for chemical analysis and dating. This paper presents the preliminary findings of the research, which include more than 600 forms of rock art found at the site.
\end{abstract}

\section{INTRODUCTION}

This paper presents the key finds from a 2009 investigation into the Gua Tambun rock art site. Gua Tambun ('Tambun Cave') is a rock shelter on the northwest side of Gunong Panjang ('The Long Mountain'), located in the state of Perak in Peninsular Malaysia (Figure 1). The shelter is located at an elevation of 50 metres and commands a panoramic view of Ipoh, the capital city of Perak. The rock art was first reported by Matthews (1959), following an investigation that same year by a team from the National Museum. Matthews' brief reports form the bulk of the current knowledge about the site, from the site's modern 'discovery' by a British military officer, to an overview of excavations carried out at parts of the rock shelter, and finally preliminary interpretations of some of the animal forms painted on the walls of the shelter. These forms identified with $\mathrm{x}$-ray detail, include paintings of 'tapir', 'dugong', and 'deer' (Matthews, 1959; 1960). The site was revisited again in 1984-1985 by another team from the National Museum, (Kam, 1985; Faulstich, 1984) who reported the discovery of several chunks of red and orangecoloured haematite, the likely material from which the paintings were created. The team also found a cord-marked pottery sherd, which was used to date the site to the Neolithic. By this time, the site was gazetted as a protected site under the Antiquities Act 1976, and access to the public was facilitated by the construction of a staircase. Anecdotal evidence suggests that the site is frequently visited by school groups, and otherwise unfrequented by the local population.

\section{FIELDWORK IN 2009}

The purpose of this study was to establish a baseline recording of the rock art and reassess some of Matthews' and other earlier researchers' assumptions. Such a reassessment was timely because of significant advances in rock art research methodology - in particular, the popularity and costeffectiveness of digital photography as a recording medium and the ability to study small samples of pigment through compositional analysis. Also, the rock art is subject to ongoing natural deterioration, and some paintings that were recorded 50 years ago can no longer be seen today. A major portion of this study involved the creation of a detailed inventory of the rock art at the site, an investigation of the pigments, reinterpretation of the site in an archaeological context. This paper will discuss the preliminary findings from Gua Tambun fieldwork in 2009, and describe the rock art forms recorded from the site.

The main recording of the rock art was carried out in January 2009; during the two-week field investigation, a scaffold was built over the main concentration of paintings and each rock art element was recorded using paper forms and digital photography. Digital enhancement and analysis of the photographs were later used as a non-invasive way of restoring faded paintings and taking measurements of the rock art. In addition, samples of the rock paints were collected from various paintings for laboratory analysis and 


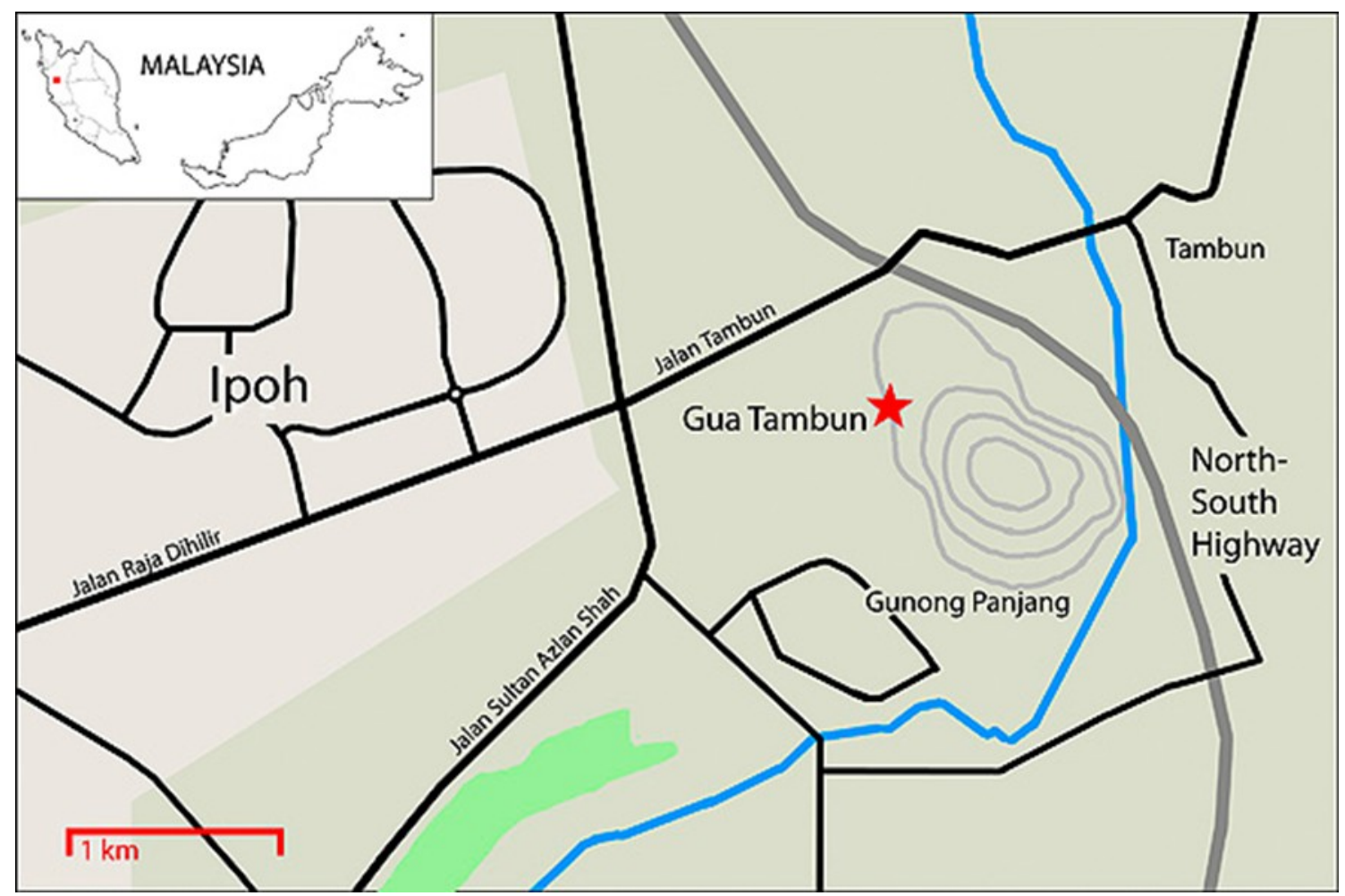

Figure 1. Location of Gua Tambun.

identification. A surface survey yielded some artefacts and ecofacts, including a stained grinding tool fragment and lumps of haematite. However, the provenance of these finds is problematic, as the surface of the rock shelter is highly disturbed by small-scale mining and visitor activity. The site was revisited several times through the year to gather supplementary data, as well as to record faded paintings that were discovered after digital enhancement.

\section{DESCRIPTION OF SITE AND ROCK ART}

Gua Tambun is more accurately described as a rock shelter rather than a cave, as it is a ledge located 50 metres above the floor. The 100-metre-long shelter runs northwest to southeast before gently curving to the south. Access to the shelter is facilitated by a concrete staircase leading to the northern end of the shelter, although it is not known if this location was the main access point during the painting period since the elevation is quite steep; Matthews' 1959 team entered the shelter closer to the centre after cutting a path through the jungle slope. The paintings are located along the cliff face, but are mostly distributed on the northern half of the cave and concentrated near the centre of the shelter. (Figure 2).

In the southern part of the shelter, where there are few paintings, the floor is relatively undisturbed and slopes steeply to the cave wall. The floor at the northern part of the shelter is generally uneven due to human activity. Matthews reported that cultivators had previously mined the shelter for guano, and Matthews himself had excavated in this area. In addition, the shelter has been open to the public since the 1980s. As a result, the floor is strewn with detritus and fluctuates between two and three metres in depth. In this disturbed section the original floor may have been roughly one metre higher than it is today.

At the start of fieldwork, four panels of rock art were known to researchers. They were labelled A-D from north to south, with the main panel of paintings is designated as Panel C. These panels are generally well-preserved, and Panels A$\mathrm{C}$ occur at least seven metres above the floor. Panel $\mathrm{D}$ is located just above the former floor level, up a steep rocky slope, and can be reached by determined climbers. During fieldwork, additional panels were documented and were designated Panels E-K in order of their recording. These later panels consist mainly of smaller clusters of rock art that are easily overlooked. They were first reported in an earlier paper (Tan and Chia, 2010). Each of these panels will be described briefly in turn.

\section{Panel $A$}

Located near the entry staircase to the site, Panel A (Figure 3 ) is a small collection of paintings located seven metres above the floor; it is often overlooked due to its size. The set of paintings can be described as a row of four 'exclamation 


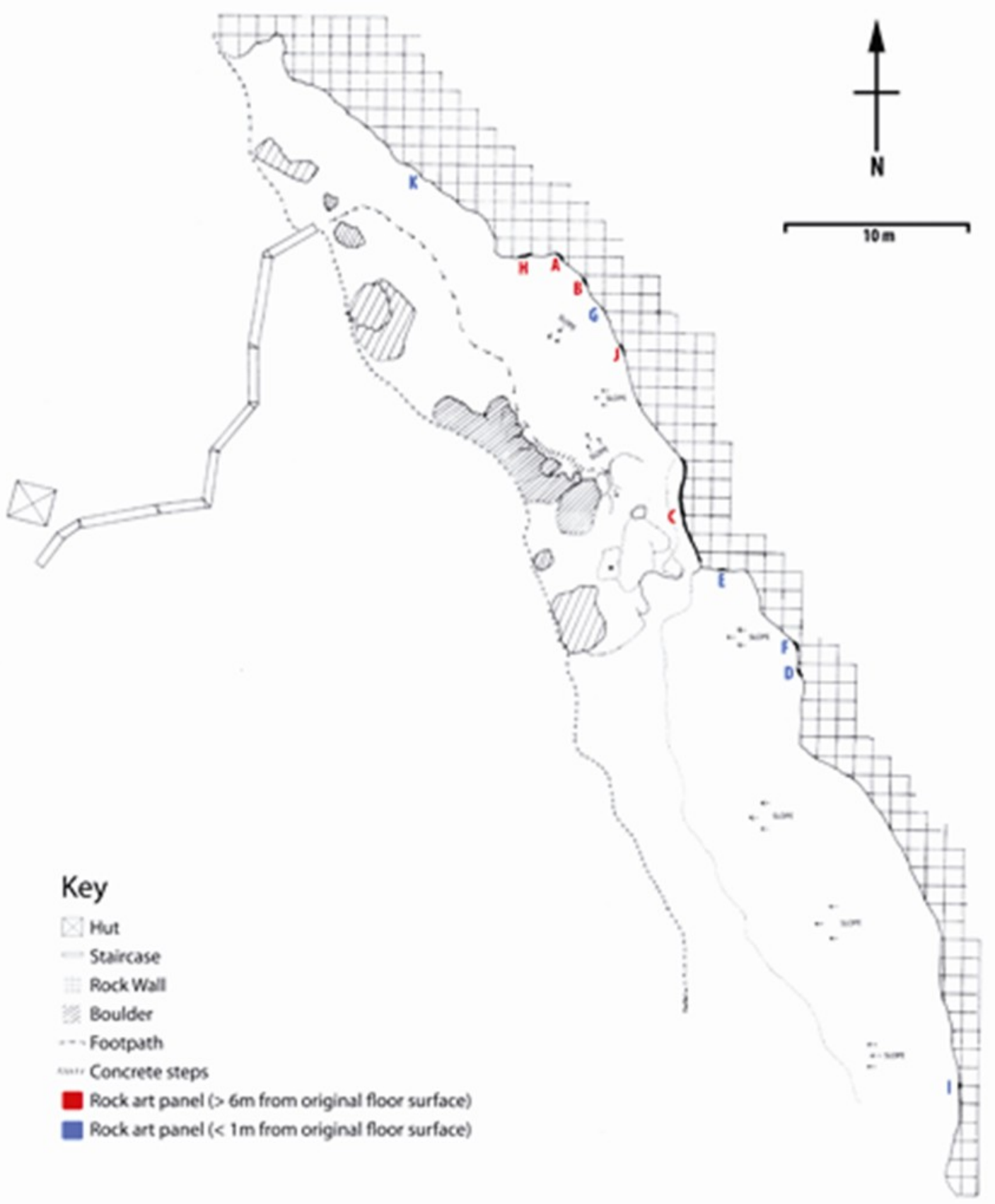

Figure 2. Map of Gua Tambun and location of paintings.

points' painted in a deep red colour. Digital analysis reveals an earlier layer of faded red figures painted underneath the 'exclamation points' that are barely visible to the naked eye. A white patina appears to have developed over the paintings. This panel appears to be relatively pristine and may not have been touched by any research team. As such, it has great potential for future geochemical research.

\section{Panel B}

The paintings in Panel B are the highest set of paintings on the shelter wall, both in absolute terms and in relation to the other panels. This panel is situated approximately 25 metres above the floor, a height that is mitigated by the deep depression in the floor below it. Because of its height, and the lack of suitable vantage points, recording this panel was particularly challenging. The prominent elements of Panel B 
BULLETIN OF THE INDO-PACIFIC PREHISTORY ASSOCIATION 31, 2011

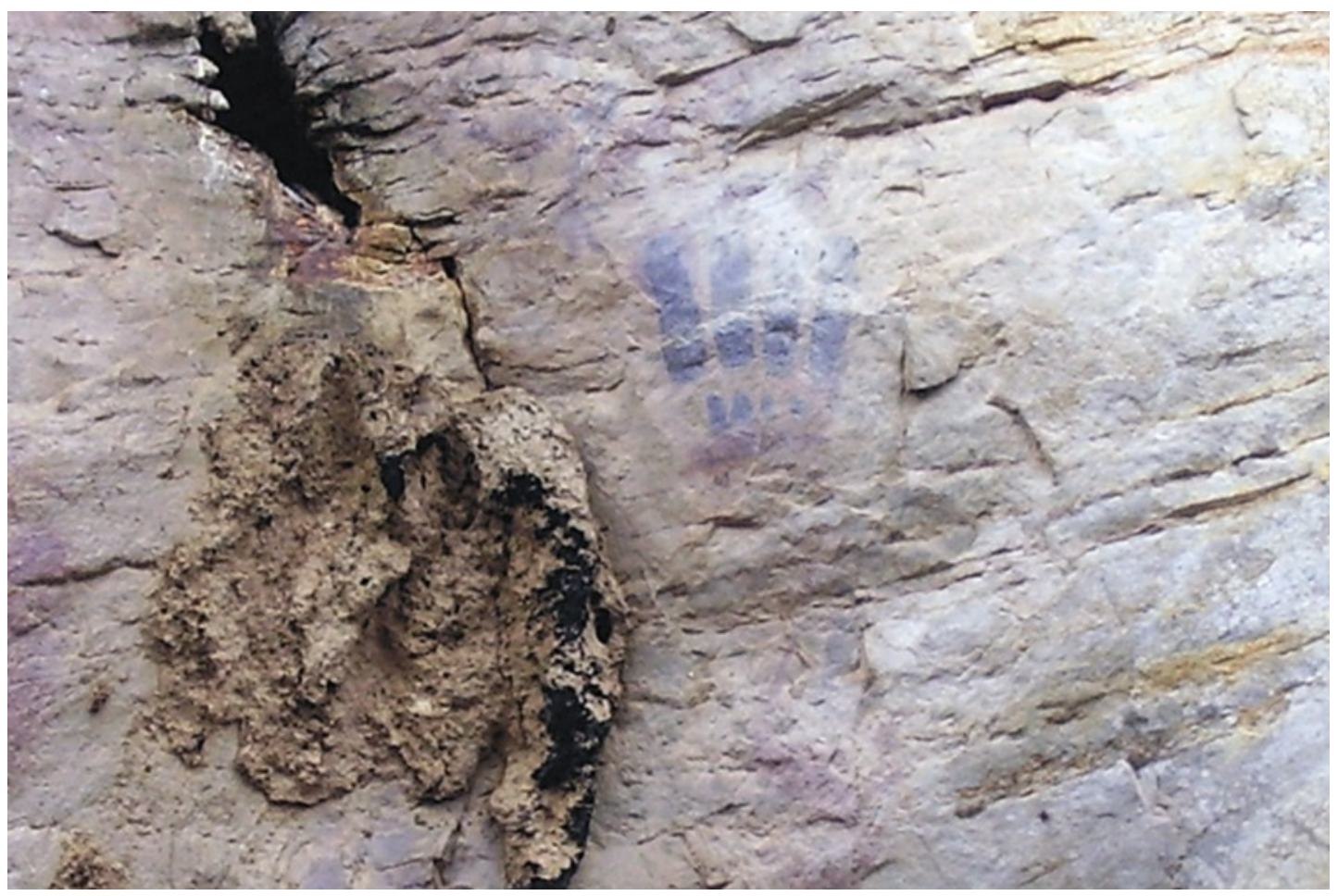

Figure 3. Panel A.

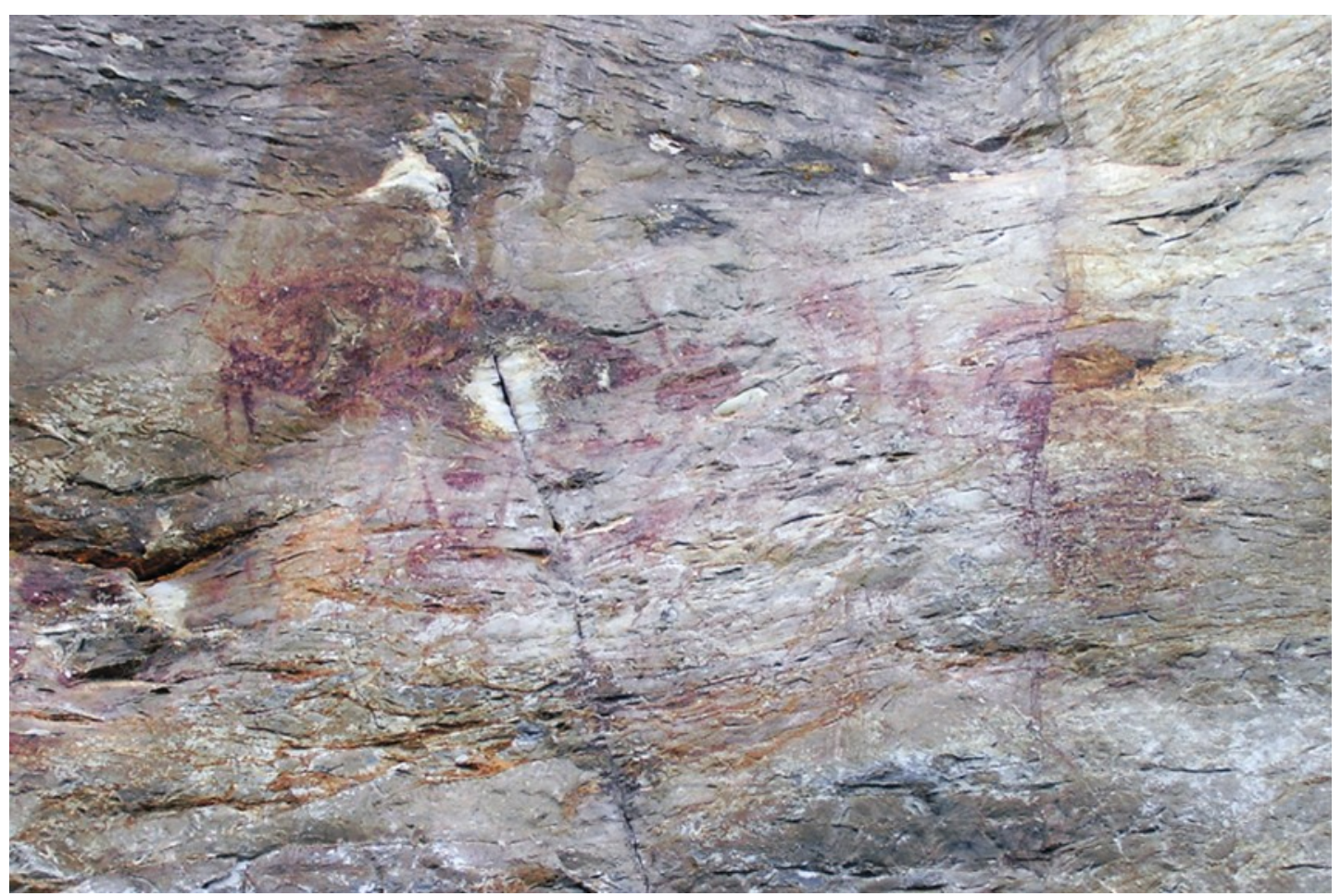

Figure 4. Panel B. 
TAN AND CHIA: CURRENT RESEARCH ON ROCK ART AT GUA TAMBUN

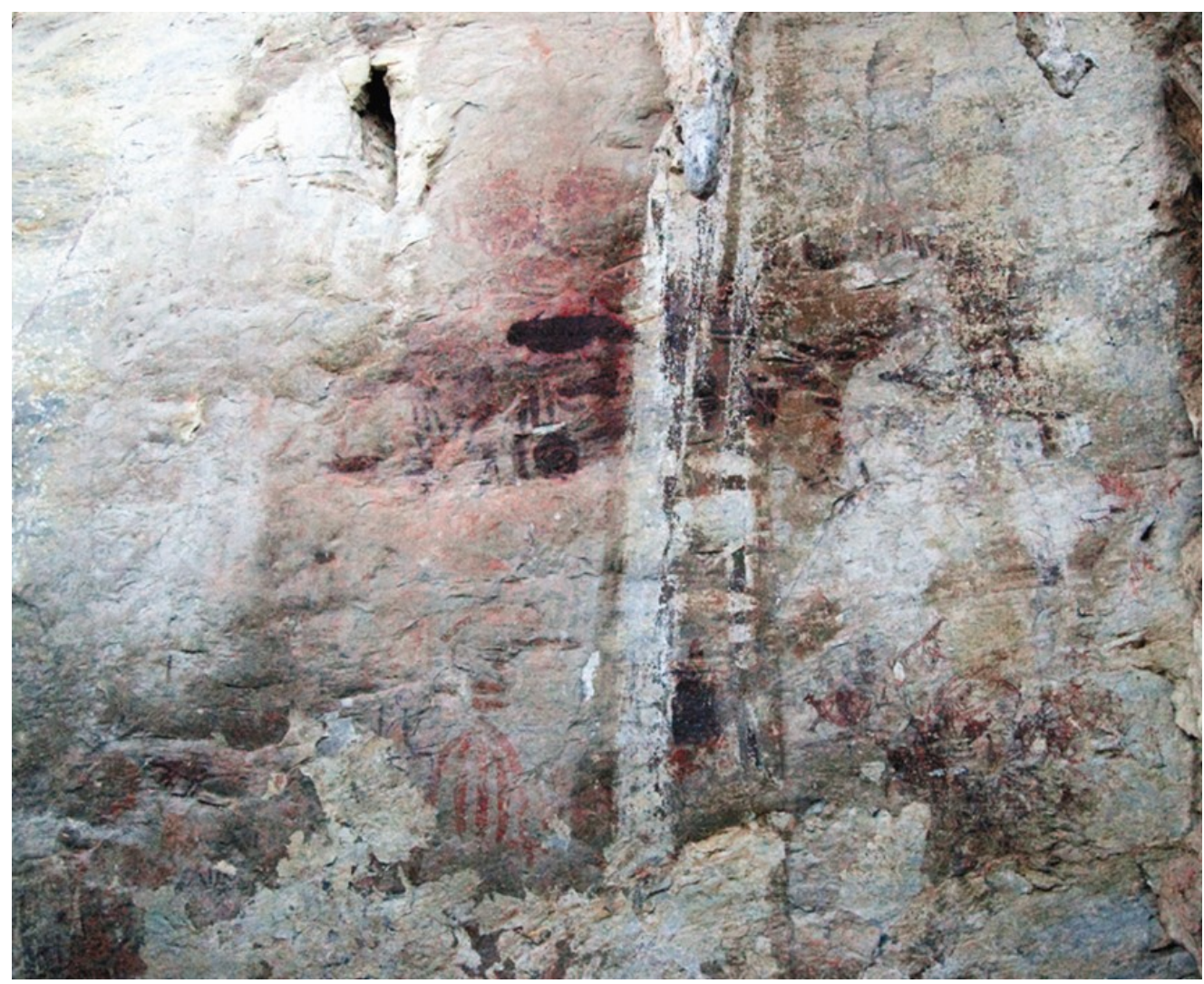

Figure 5. Panel C.

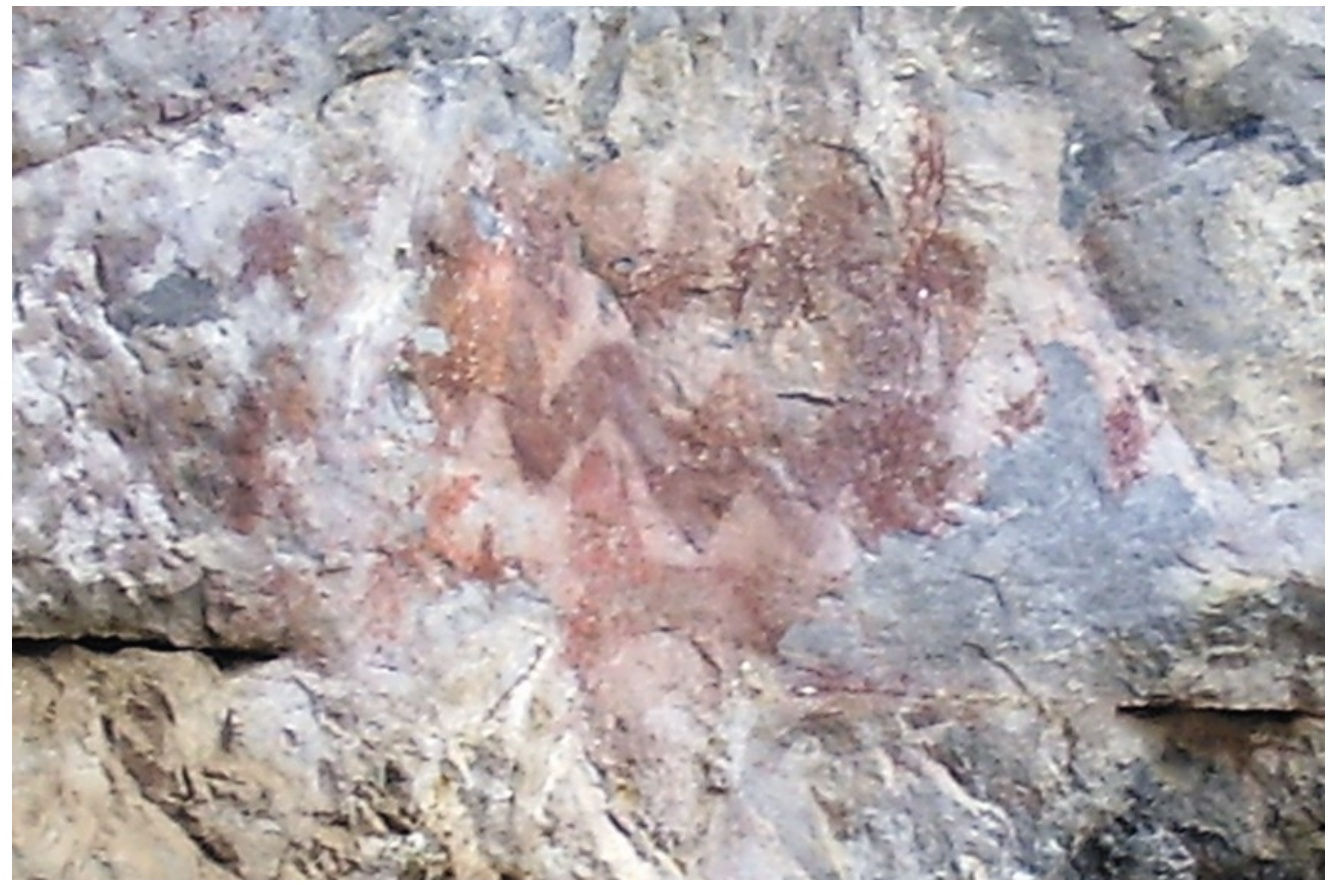

Figure 6. Panel D. 


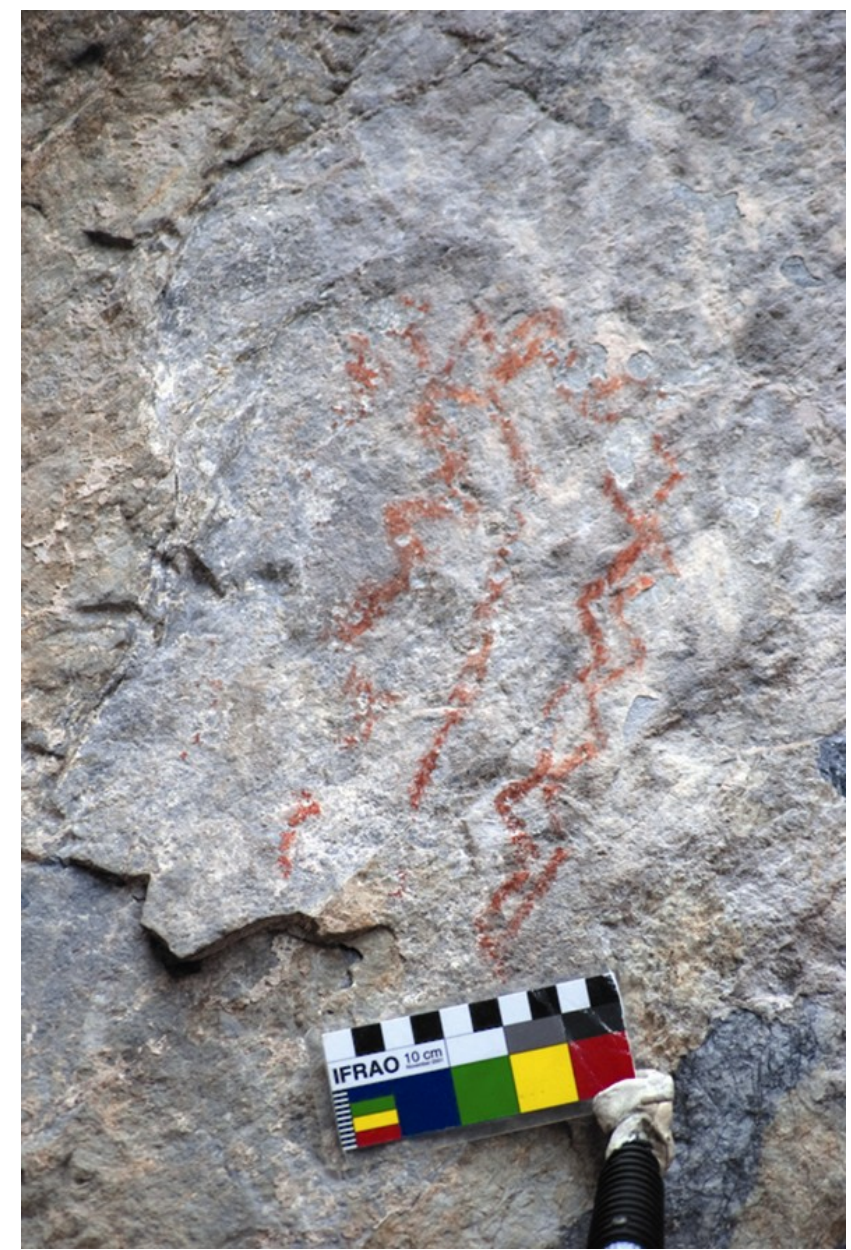

Figure 7. Panel E.

(Figure 4) are a depiction of a four-legged zoomorph, possibly a 'wild pig' surrounded by anthropomorphs depicted with round bodies, thin legs and upraised arms. Besides depictions of other smaller zoomorphs, the scene also contains what may be depictions of 'pots' hung on sticks, which may lend credence to the proposed Neolithic date of the site. However these 'pots' could just as easily be interpreted as 'fruit'. Many portions of this panel appear to have weathered significantly from surface exfoliation and water wash. Because of its extreme height and inaccessibility, this panel also does not appear to have been accessed by researchers in modern times and has potential for future study.

\section{Panel C}

The largest concentration of rock paintings in Gua Tambun is contained in a space some six metres above the floor of the shelter. It is approximately 10 metres wide and 10 metres high (Figure 5). This is the most prominent panel within the shelter, and most of the iconic paintings of this panel-such as the depiction of the 'dugong', the 'x-ray style deer' and the 'dancing man'- have been featured in earlier reports. It is notable that this panel alone contains over 500 individual rock paintings. In contrast to most of the other panels, which are painted in monochrome red, the paintings here include orange, red and purple, although each element is painted in a single colour and there are no examples of individual polychromatic paintings.

A large number of these paintings are highly faded or damaged by the elements, making them hard to make out from the normal vantage point of the shelter floor. In the 1980s, a team from the National Museum in Kuala Lumpur built scaffolding to reach the paintings in Panel $\mathrm{C}$ in order to draw chalk outlines around the forms to enhance their visibility, albeit with mixed results. Water wash can be observed in the upper levels of the panel, causing much damage to the paintings; a clean vertical white stripe obscures the centre of the panel. However, it appears that the wash is old; it appears in Matthews' 1959 pictures of the site. Unfortunately, the paintings above the 'dugong' have deteriorated significantly in the last 50 years, as they were sketched by Knuth (1962). Today, they are extremely faded and cannot be recognised. Much of the lower section of the panel appears to have been damaged by heavy exfoliation, possibly due to mining activity. This panel also displays a complex array of superimpositions which may represent different periods of painting, and we were able to distinguish at least seven distinct painting periods based on the superimpositions, and in one case, the distinctiveness of the 'x-ray' art.

\section{Panel D}

Situated some 10 metres south of Panel C, Panel D (Figure 6) represents a significant departure from the previous panels, as it is fairly close to the floor and not elevated. Unlike the earlier panels, the paintings on Panel D seem to be composed primarily of dark red linear designs--mainly thick horizontal wavy lines and thin vertical wavy lines--which do not appear in the paintings at the higher elevations. The paintings appear to be stained by water, causing the edges to bleed into the background rock and making the shapes more difficult to determine.

\section{Panel E}

Panel E (Figure 7) is a small set of paintings located between Panels $\mathrm{C}$ and $\mathrm{D}$ near to the original surface line. The painting is a linear design consisting of fine wavy vertical lines in red colour, and may be related to a similar design found on Panel D. 


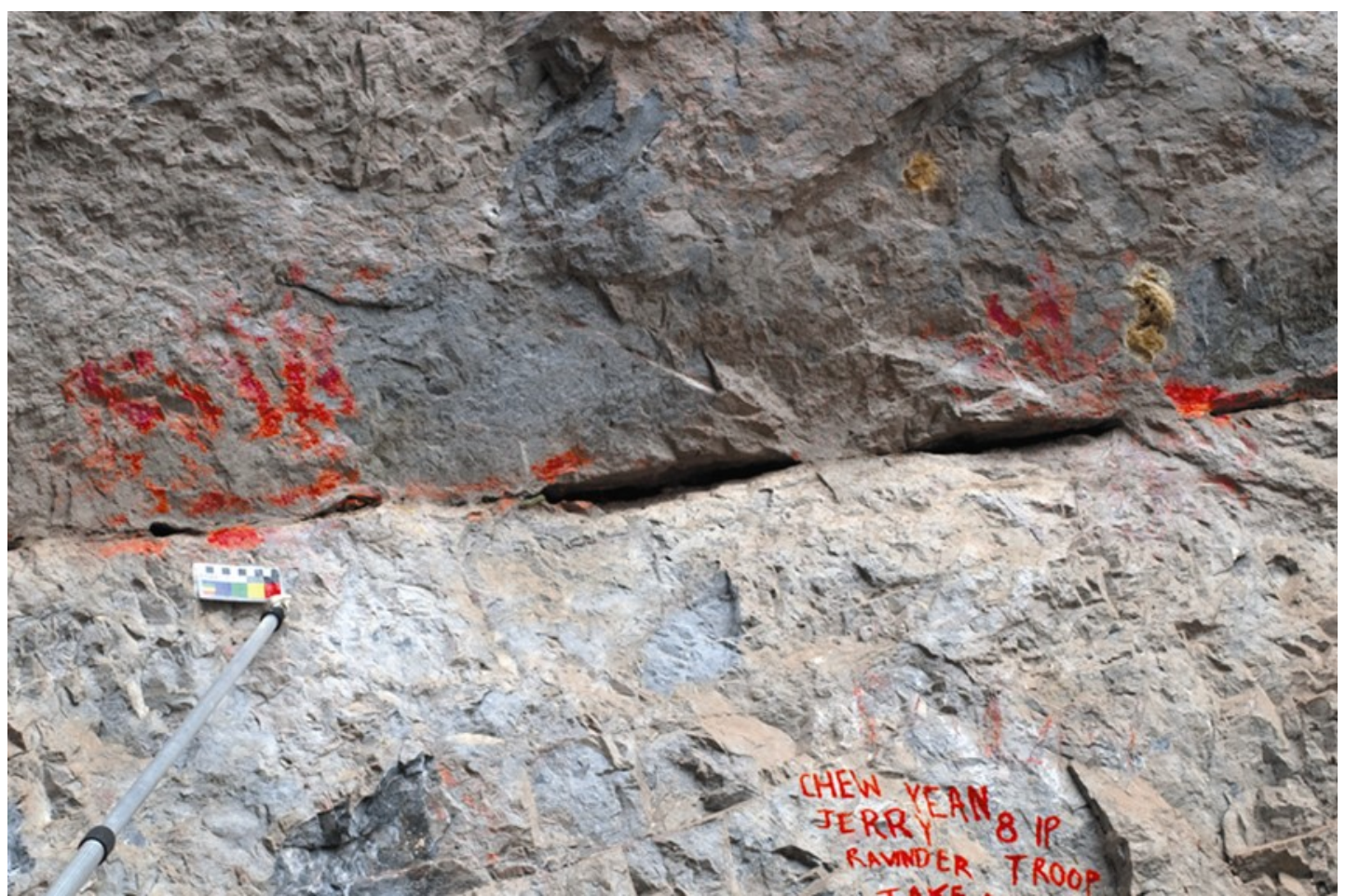

Figure 8. Panel F.

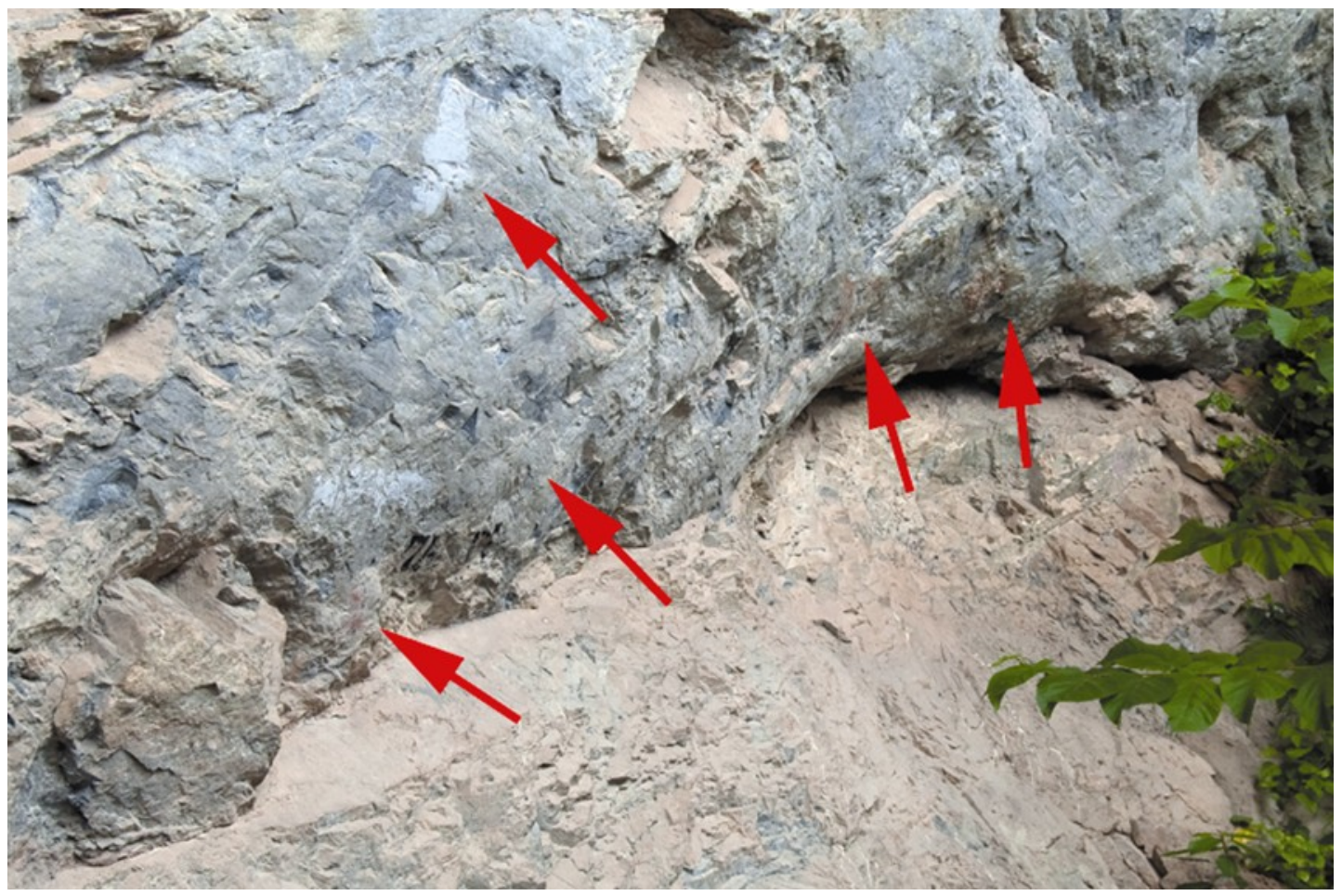

Figure 9. Panel G. 


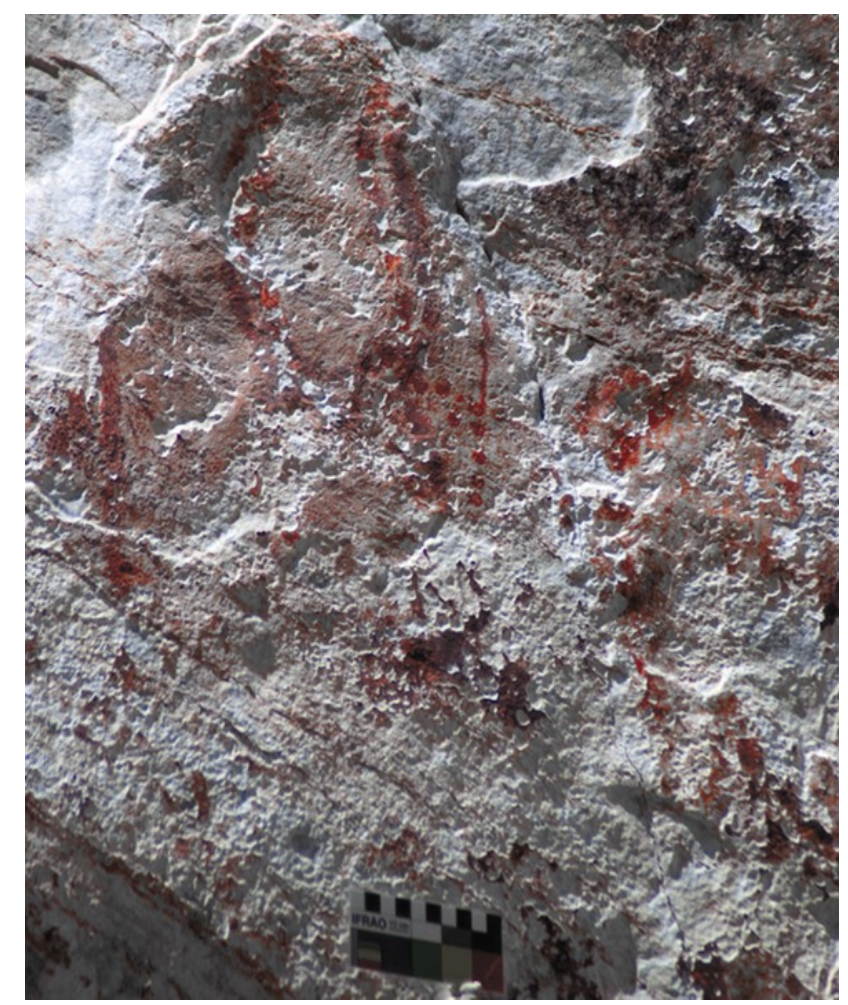

Figure 10. Panel $\mathrm{H}$.

\section{Panel F}

The painted forms of Panel F (Figure 8), situated between Panels D and E, appear to be heavily damaged by water wash; consequently, the paintings are indistinct. The unnatural red staining, clustered at two spots, suggests that something might have been painted on the rock wall, which seems to have been damaged in a similar fashion to the lower section of Panel C.

\section{Panel $G$}

Panel G (Figure 9) is a five-metre stretch of wall just above the floor between Panel B and Panel J, located in front of the area excavated by Matthews in 1959. The majority of the paintings are small linear drawings, but two paintings stand out. The first is an anthropomorphic form on the southern side depicting a frontal-view anthropomorph wearing 'earrings' and a 'topknot'; it does not appear to have any similarity with other anthropomorphic forms in the site. A second form, also an anthropomorph, in the northern end of the panel, is notable as it has been painted over by a layer of grey paint, presumably as an effort to erase modern vandalism. It seems that whoever painted over the vandalism may have also erroneously painted over an authentic rock painting. The rest of the paintings in this panel are linear abstract designs and do not appear to be related to one another. The variety of forms and subtle colour differences indicate that they may have been painted at different points of time.

\section{Panel H}

Another small set of paintings (Panel H, Figure 10) was located seven metres to the north of Panel A. Panel $\mathrm{H}$ is heavily damaged by spalling and exfoliation, and none of the original forms of the paintings can be determined, although it appears that the panel contains both red and purple-coloured paintings. Some finger dots can be identified in one of the paintings.

\section{Panel I}

The lone rock art panel located at the southern end of the rock shelter is intriguing for a number of reasons. Panel I (Figure 11) is unusual because it is painted in orange, a colour which is otherwise only found on Panel $\mathrm{C}$, but unlike Panel C is located at floor-level. The panel contains six oval shapes painted as solid-silhouettes which are similar to the paintings in the elevated panels.

\section{Panel J}

Appearing as white figures over a blackened background, the exact nature of this panel is undetermined. The rock art of this panel look like petroglyphs, in which material is removed or carved from the original surface. However, another suggestion is that these may be the 'shadows' of former paintings, and represent advanced weathering of the pigments. Some elements in Panel $\mathrm{J}$ share some visual similarity with the rock art in the adjacent Panel C, but others appear to be unlike the rest of the rock art in Gua Tambun (Figure 12). This panel contains repetitive designs such as rows of vertical lines and rows of chevrons which are not found in other panels.

\section{Panel K}

The area around Panel K (Figure 13) was initially overlooked because of all the modern vandalism present. However, the discovery of the 'new' rock art panels very close to the floor of the shelter (E-G, I) led to a re-evaluation of this area. Four figures have been interpreted as ancient rock art on the basis that they share the red colour found predominantly in the site's rock art, as well as their similarities with the other paintings on Panels B and G.

\section{GENERAL OBSERVATION AND INTERPRETATIONS}

A total of 640 rock paintings were recorded and documented, covering a total of 27 square metres of space. These numbers were surprisingly high, considering estimates by Matthews (1960) suggested that only 80 paintings could be seen clearly, 
TAN AND CHIA: CURRENT RESEARCH ON ROCK ART AT GUA TAMBUN

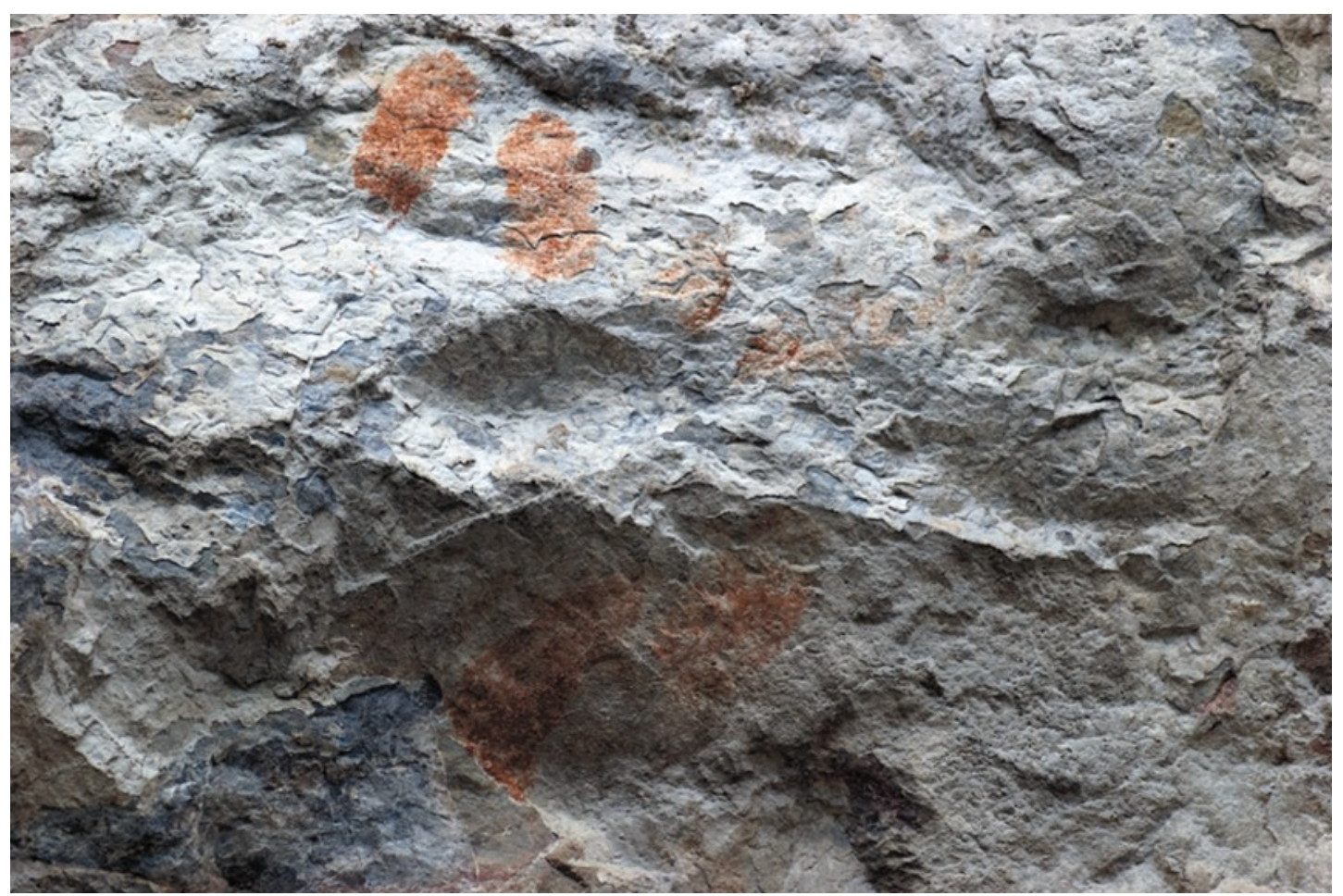

Figure 11. Panel I.

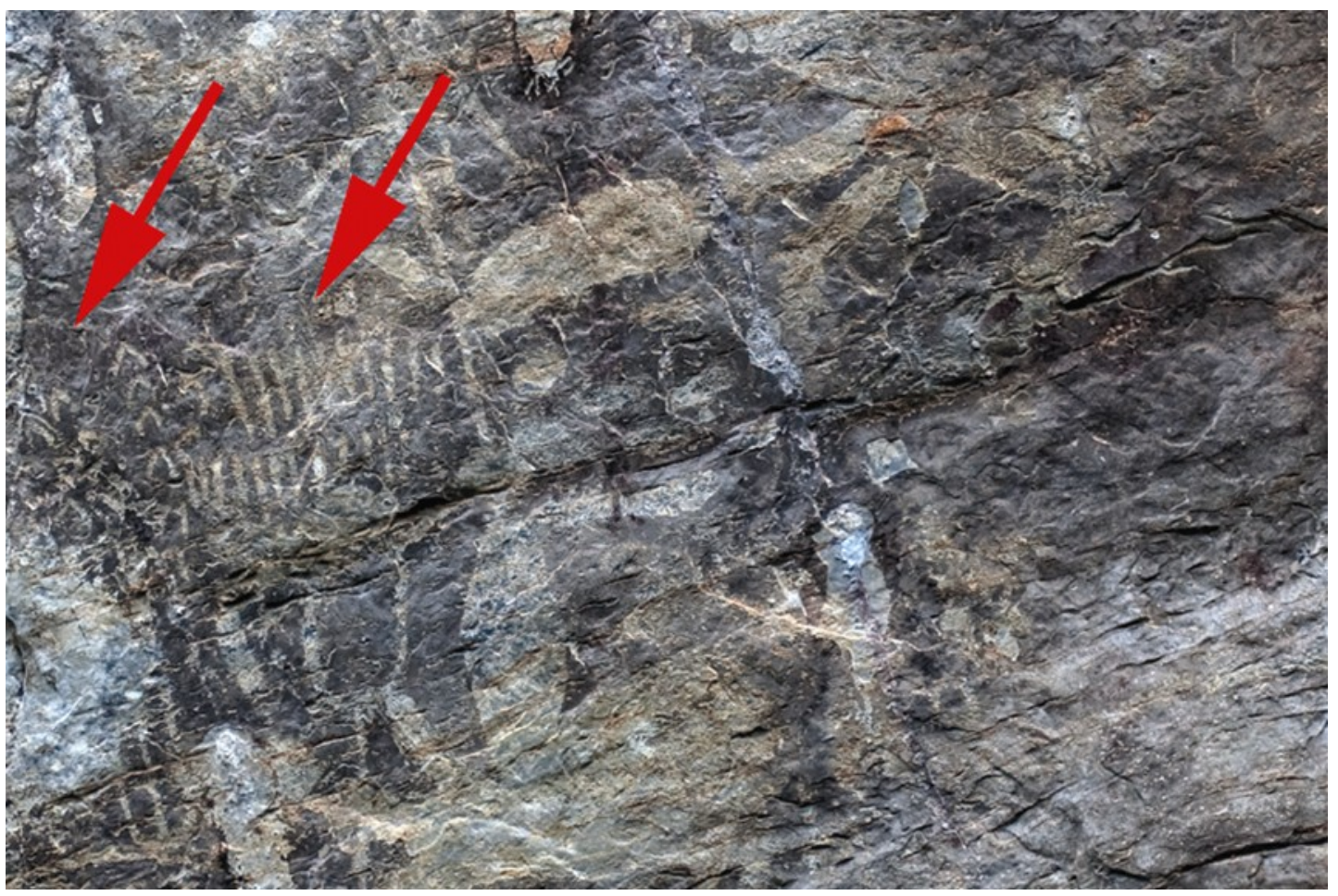

Figure 12. Panel J. 


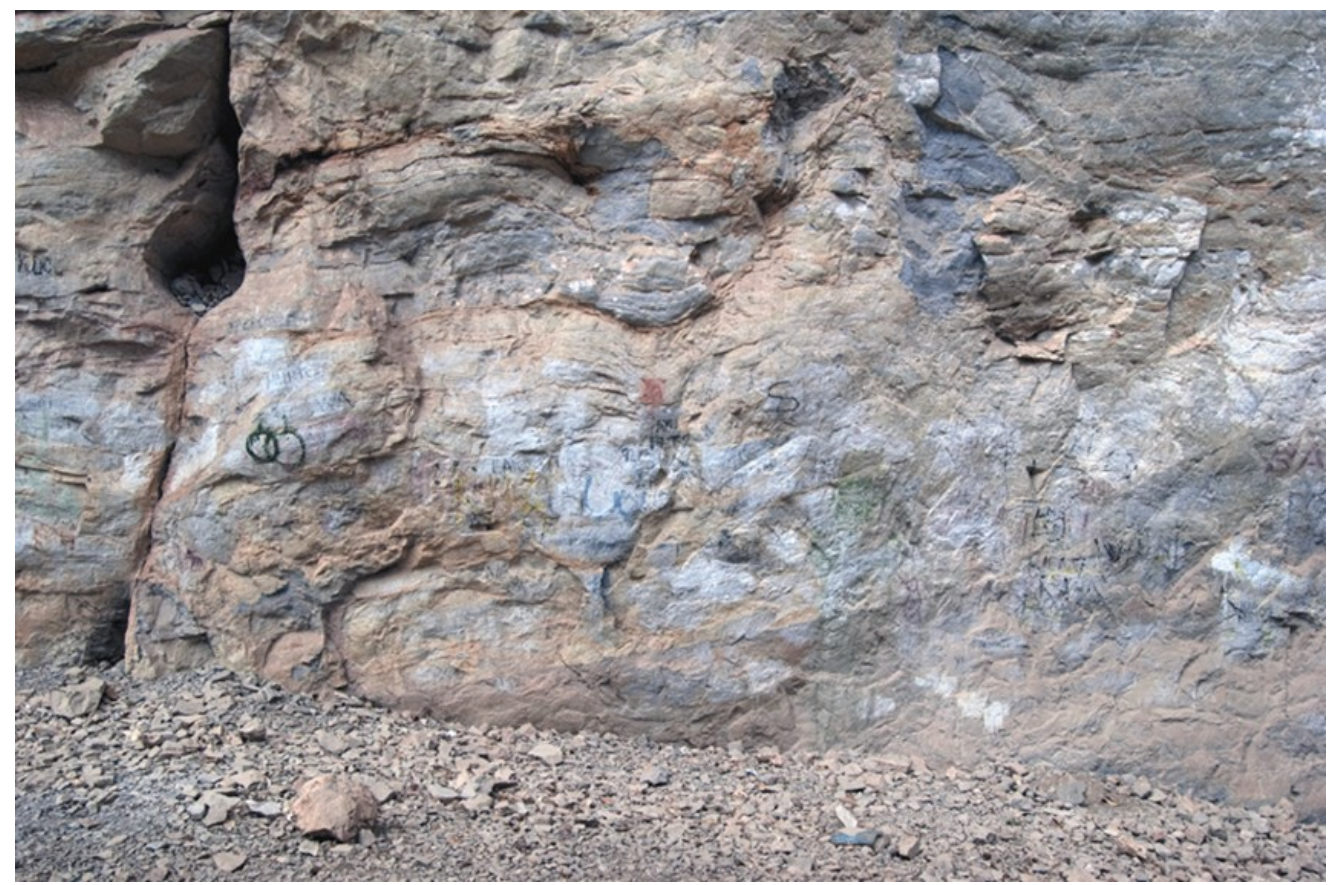

Figure 13. Panel K.

a
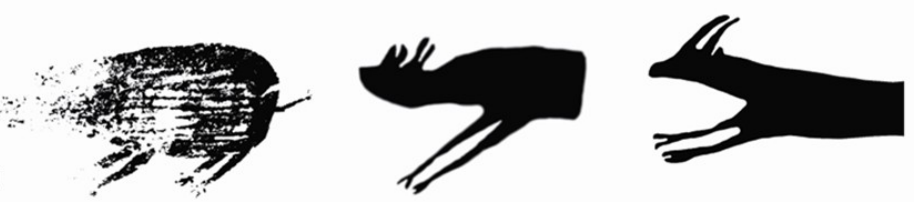

b
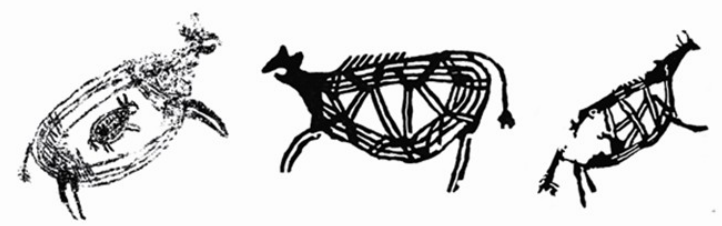

C
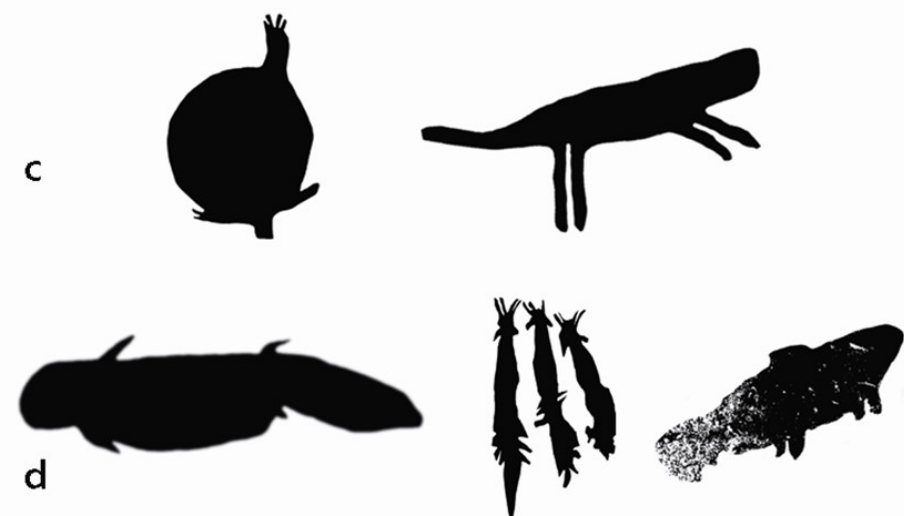

Figure 14. Zoomorphic forms found in Gua Tambun, including land animals such as 'boar' and 'deer' (a), 'mountain goat' (b), reptiles and amphibians like 'turtles' and 'lizards' (c) and aquatic creatures such as 'catfish' and various fish (d). Not to scale. 


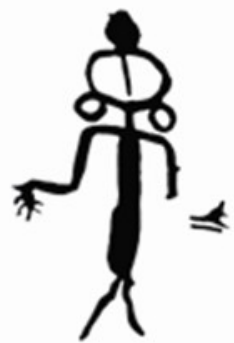

1

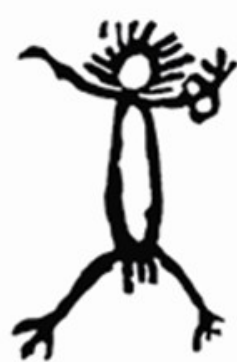

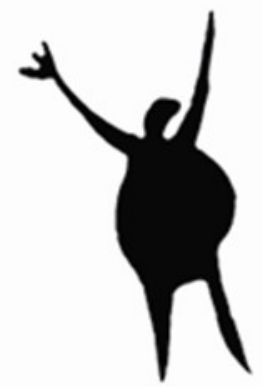

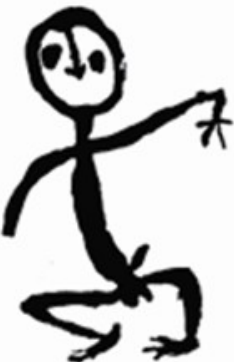

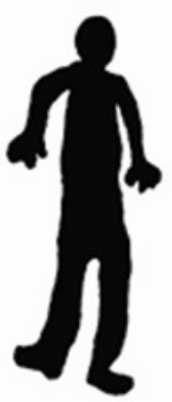

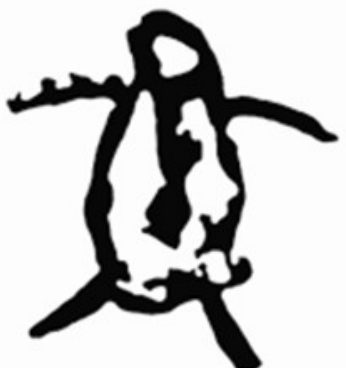

Figure 15. Anthropomorphic forms found in Gua Tambun display a high degree of variability and largely differ from one another. Not to scale.
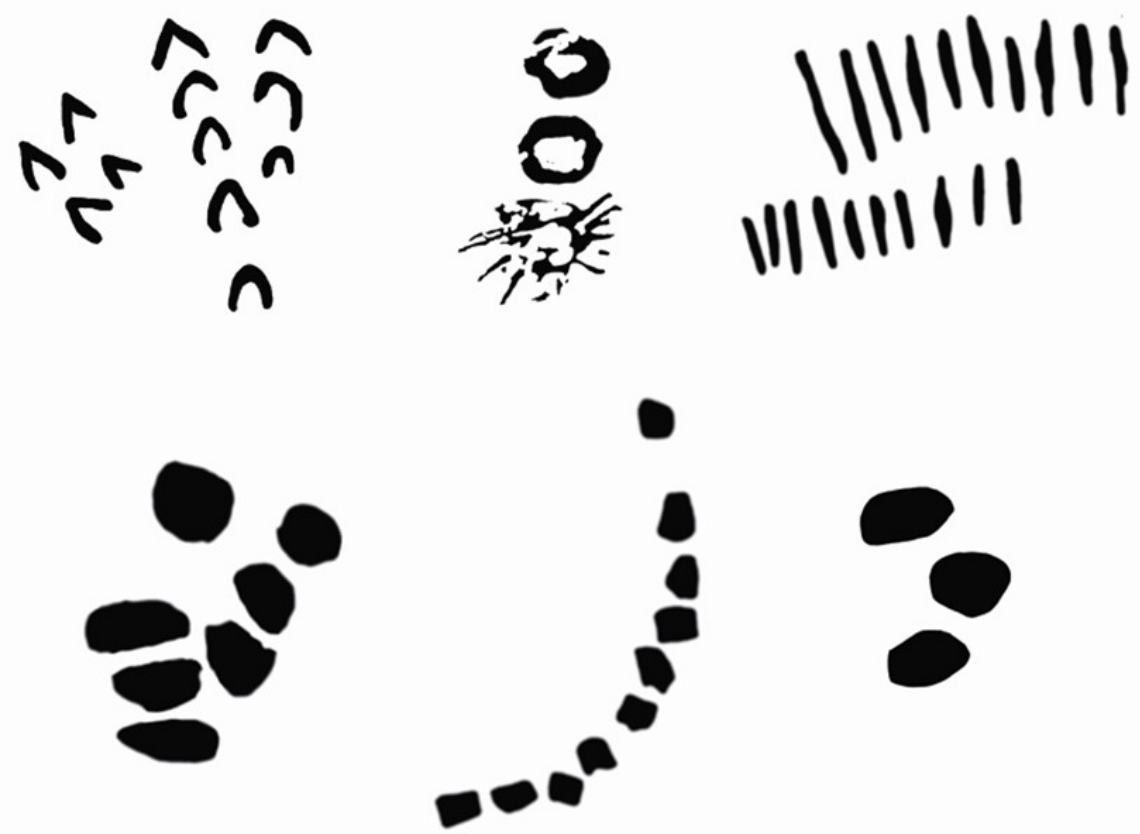

Figure 16. Geometric forms are the most plentiful type of motifs at Gua Tambun, ranging from repetitive chevron, linear round and oval shapes. Not to scale. 


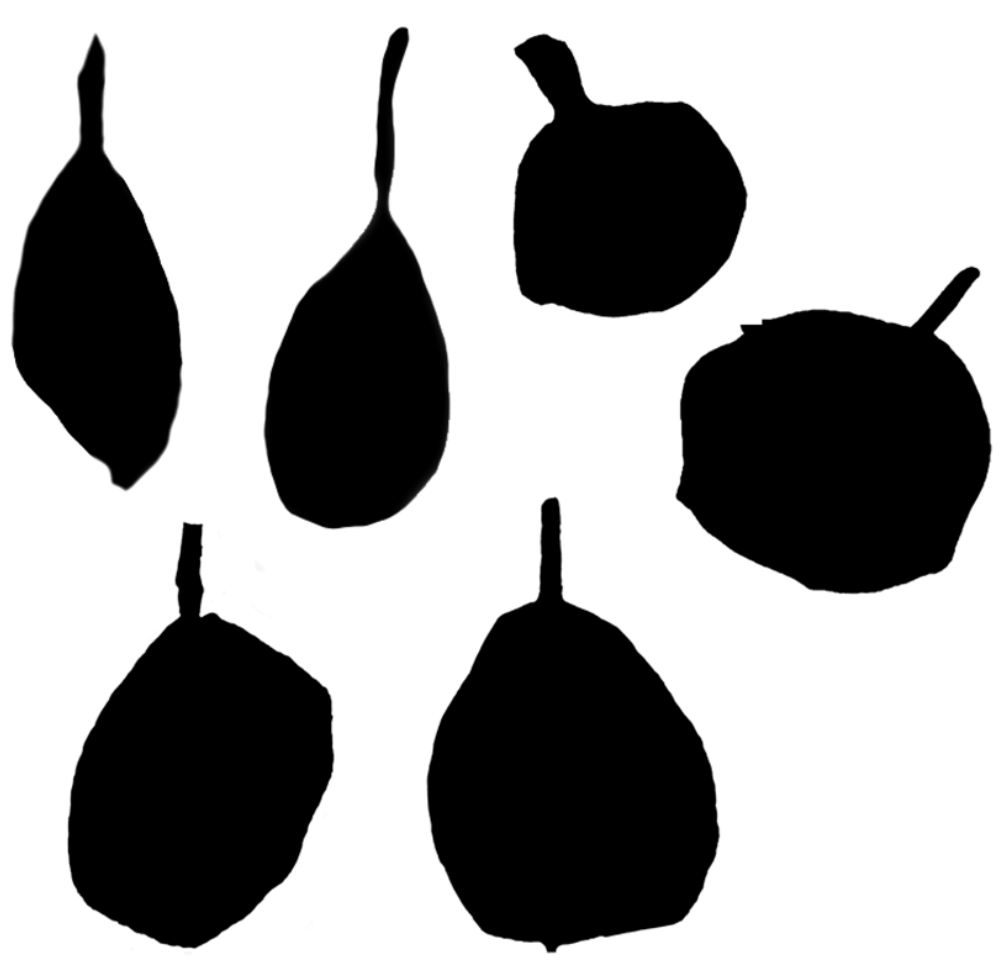

Figure 17. Botanic forms at Gua Tambun appear to be confined to these 'fruit' shapes. Not to scale.

while pamphlets distributed by the local heritage society listed an even smaller number of 20 to 30 . A significant proportion of the rock art was visualised using digital enhancement techniques that enabled us to manipulate the colours and lighting conditions of each image without modifying the actual rock art itself. The number of rock paintings recorded from Gua Tambun, as well as their scale and position relative to the floor underscores the time invested by the painters to create them.

In order to make sense of the data collected from the rock art recording, each rock art element was measured and classified according to their colour, size and type. Each rock painting was categorized into one of five basic forms: Zoomorphs, which are elements that have been interpreted as animal forms; Anthropomorphs, or human shapes, Geometric features, including regular shapes such as squares, circles, ovals and approximations thereof; Botanic shapes that looked like 'fruit'; and Abstract shapes, rock paintings that have a definite form but could not be classified into the other four categories (Figures 14-18).

The predominant type of rock art on the walls of Gua Tambun is the Geometric form, accounting for $52 \%$ of the rock art present (Figure 19). The Zoomorphic and Anthropomorphic paintings together form only $11 \%$ of the total number of rock art elements at the site; however, it should be noted that the zoomorphic forms account for over $50 \%$ of the rock art by surface area (Figure 20 ).

The proportion of surface area devoted to animal forms suggests that the zoomorphs played an important role in the minds of the prehistoric artists that painted them. With the exception of the ' $\mathrm{x}$-ray' drawings, the animal forms that have been depicted tend to be naturalistic, in silhouette, and painted in profile or in bird's-eye view. In some cases, the zoomorphs have been painted life-size. The decision to paint animals on a large scale is notable since these large depictions are located high above the floor of the shelter-at least 10 metres high, suggest that some degree of planning and deliberation on the part of the artists. Some of the interpreted animal forms include deer, wild pig and turtles. Matthews (1960) reported that the faunal remains of such animals were discovered during his 1959 excavation. The superimpositions of the paintings further suggest that the large zoomorphic paintings on the higher elevations were some of the earliest paintings created, and that over time the painters decided to move away from painting nonzoomorphic subjects and smaller-sized forms.

The depictions of anthropomorphs are also interesting, as they display a wider degree of variation in terms of sizes, 

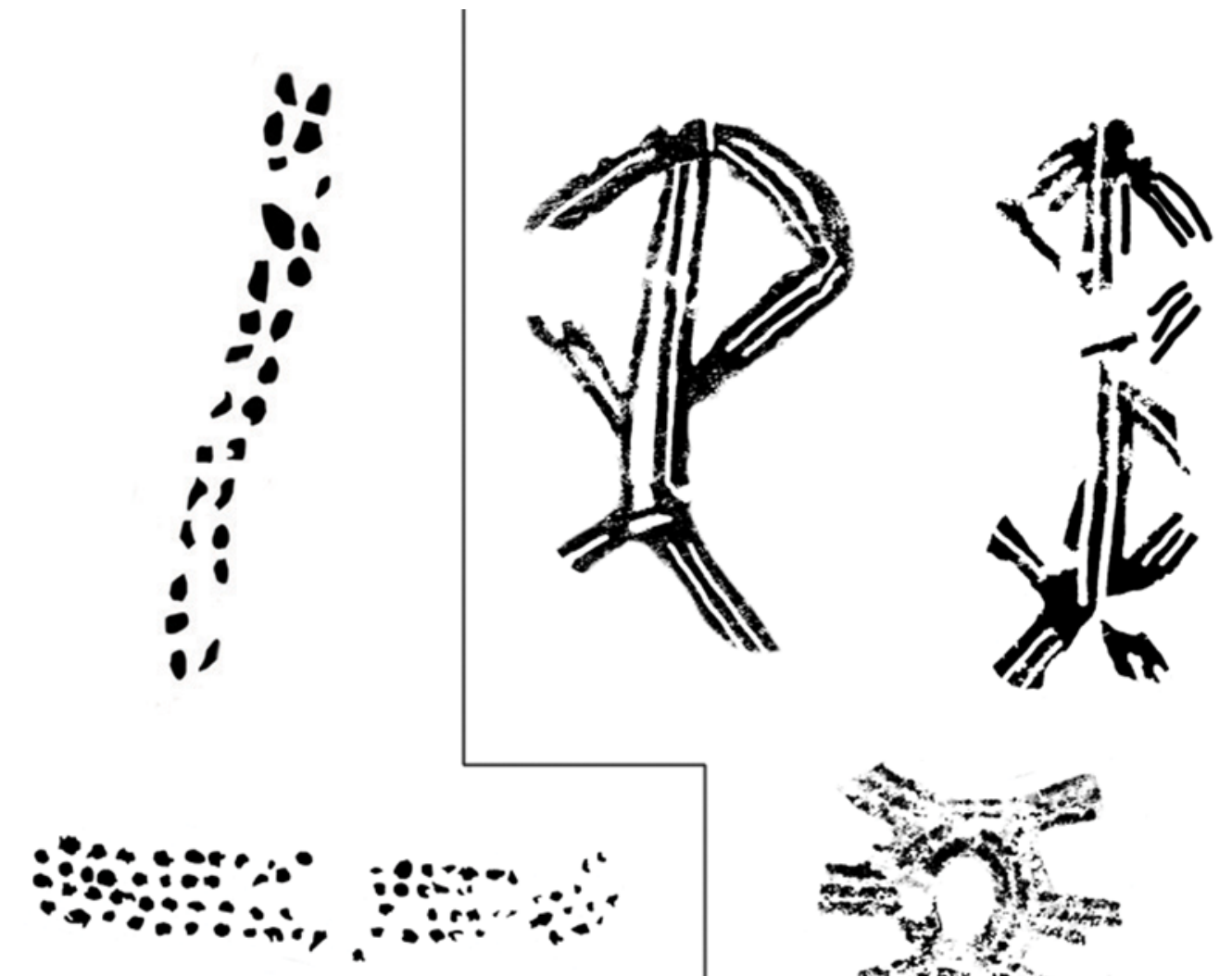

a
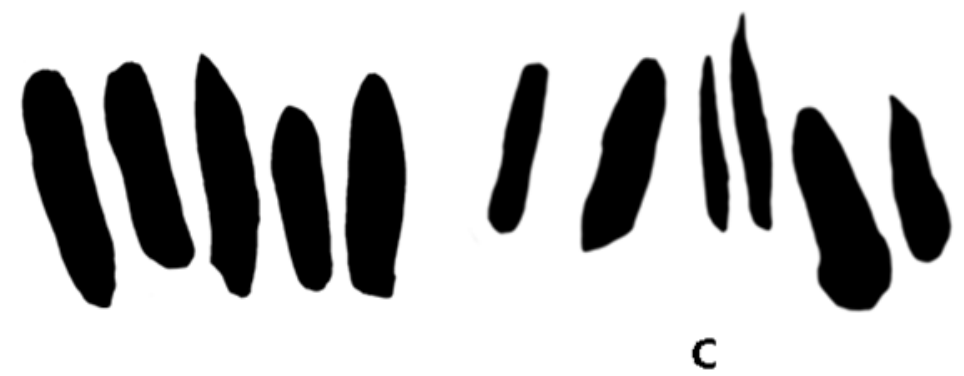

C

Figure 18. Gua Tambun contains a number of abstract motifs such as a finger dots (a), trilinear designs (b) and clusters of ovals (c). Not to scale. 


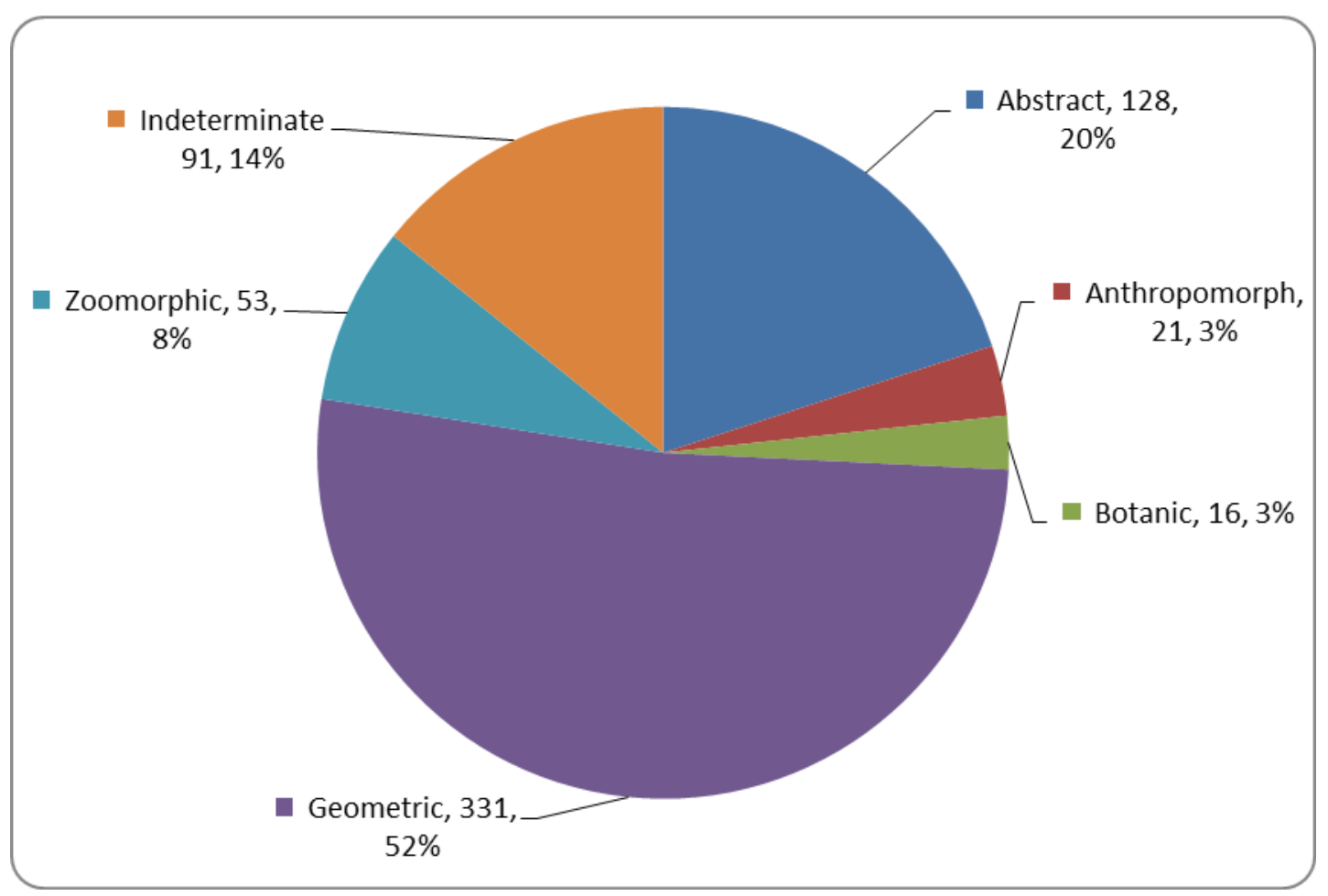

Figure 19. Distribution of Rock Art by Category

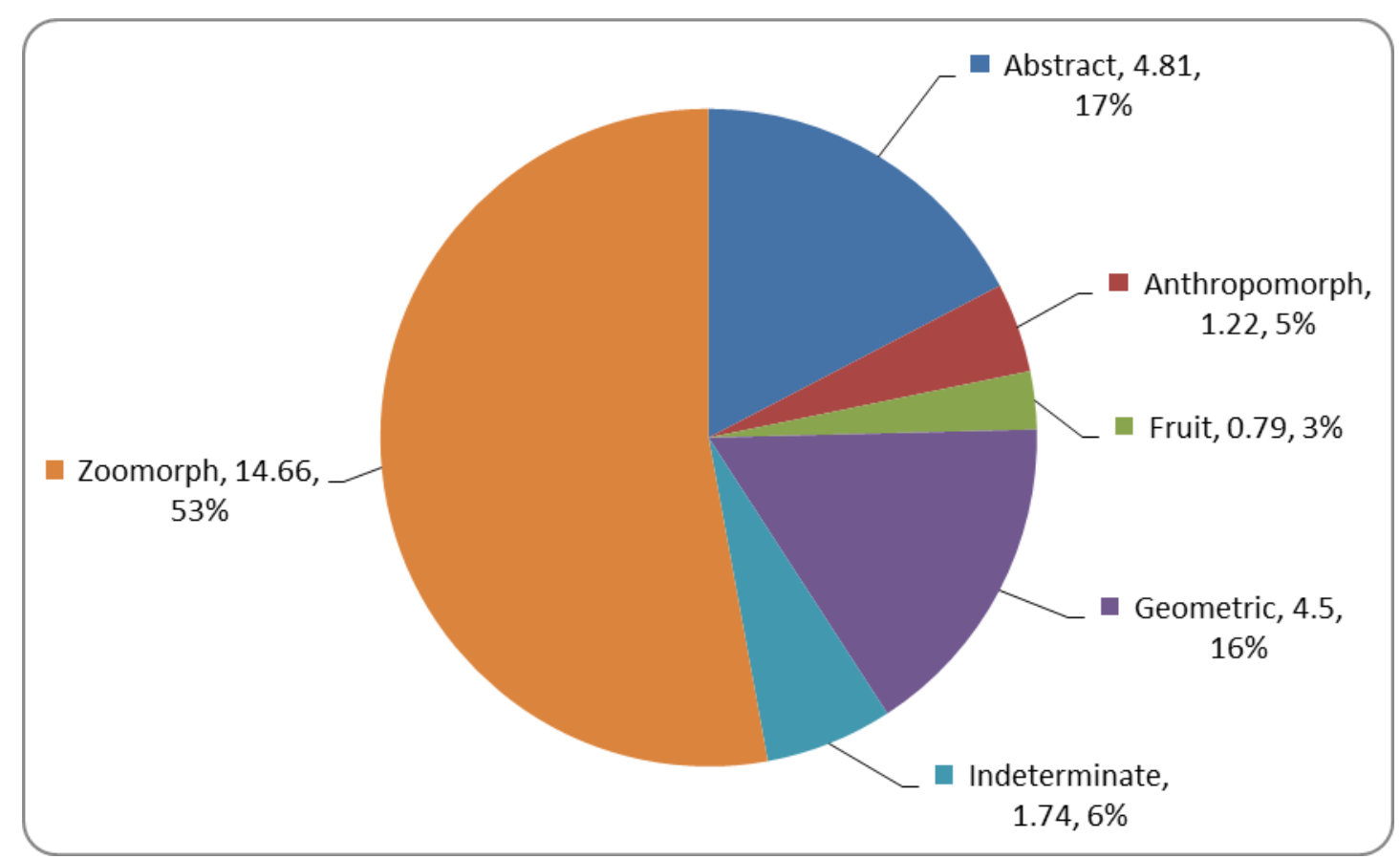

Figure 20. Distribution of Rock Art by Surface Area 
body shapes, postures and types of painting than the zoomorphs (Figure 16). Apart from the anthropomorphic forms on Panel B, which are fairly consistent, none of the anthropomorphs resemble one another. This variation in anthropomorphic forms suggest that the rock art was the work of multiple authors, or even multiple groups of authors, assuming that the depiction of self-imagery is largely consistent from culture to culture. Several depictions of anthropomorphs are seen to be wearing ornaments such as headdress and bracelets, but none of them can be used diagnostically to determine which among the indigenous populations of the Malaysian peninsula may have been responsible for their creation. The variation of depictions, their size, and their various locations throughout the rock shelter also indicate that the site was in use over a long period of time, perhaps for different purposes and by different artists or groups of artists.

The paintings on the main panel, $\mathrm{C}$, appear in three colours--red orange and purple--each with their own varying degrees of saturation and fading, while most of the other panels are monochromatic and predominantly red in colour. Analysis of the pigment samples using x-ray diffraction and scanning electron microscopy confirmed the earlier assumptions that the rock art were made using haematite, which was likely drawn from local sources. The three main colours observed on the rock art were accounted for: purple colours were probably derived from powdered iron ore, abundant around the foot of Gunong Panjang, while the red and orange haematite reportedly found in situ by earlier researchers (Faulstich, personal communication, July 24, 2009), accounted for their corresponding colours. Because of their inaccessibility and rarity, the white paintings from Panel $\mathrm{J}$ and two yellow paintings in Panel $\mathrm{C}$ were not tested and their exact nature is as yet undetermined.

Evidence of paint splatter in the middle of Panel $\mathrm{C}$ indicates that the pigments must have been applied in liquid form, rather than being drawn like a crayon straight onto the rock surface. It is likely that such haematite paints would be a simple mixture of ground iron ore or haematite with water. From a closer look at the rock art, it can be inferred that fingers were used to paint some of the figures on Gua Tambun, especially line art and finger dots. In some specific cases, tools must have been used in order to produce the fine lines and sharp points observed in some forms. There is no clue as to what kind of tools may have been used, and it is highly probable that such tools, especially those made from organic materials, would not survive the archaeological record.

\section{CONCLUSION}

The creation of an inventory of the rock art at Gua Tambun plays an important role in future conservation and research efforts at the site, as it provides a baseline recording from which comparisons can be made. This new data from Gua Tambun reveals new insights into the complexity of the rock art located at the site. First, the actual number of paintings recorded during the course of the research far exceeds earlier assumptions, in part due to the fact that there was little detailed work carried out at the site since its initial 'discovery' in 1959, but also because a substantial number of rock paintings were only visible when the rock wall was inspected up close. In addition, more paintings were discovered when field recordings were enhanced using digital image analysis with a computer. Coupled with sections of wall that are now irrevocably damaged, Gua Tambun is certainly the largest rock art site in Peninsular Malaysia. While the specific meanings of the paintings and what they were meant to convey may never be recovered, the deliberate placement of paintings at high elevations as well as the differences in subject matter between distinct phases of painting infer a degree of planning and deliberation in their creation.

The extensiveness of the Gua Tambun rock art also carries larger implications for site management and conservation. It has long been thought that the site would require minimal monitoring and maintenance since the rock art (Panel C) was located far above the reach of human hands. This research has demonstrated that a significant number of rock art exists at floor level, and vandalism has already encroached on some of the floor-level rock art. Thus, a new conservation and visitor management strategy must be considered in order to protect these paintings from further harm.

\section{ACKNOWLEDGEMENTS}

The authors would like to acknowledge the support of Universiti Sains Malaysia for this research, through the USM Short-Term Grant, the USM Fellowship Scheme, the USM Postgraduate Research Scheme. . Financial support to present this paper was mainly supported by the USM Short-Term Grant, with partial support from the IPPA Congress 2009 Committee.

\section{REFERENCES}

Faulstich, P. 1984. Preliminary report on the rock art of Ipoh, Malaysia. Rock Art Research 1: 141-142.

Kam, J. 1985. Tambun rock art for posterity? New Straits Times, 29 April, p. 15.

Knuth, E. 1962. The oracle at Tambun: Malay and Thai paintings compared. Malaysia in History 8: 3-10.

Matthews, J. M. 1959. Rock paintings near Ipoh. Malaya in History 5(2): 22-25. 
Matthews, J. M. 1960. A note on the rock paintings recently discovered near Ipoh, Perak. Man 60: 1-3.

Tan, N. H., and S. Chia. 2010. 'New' Rock Art from Gua Tambun, Perak, Malaysia. Rock Art Research 27(1): 918. 